\title{
La dramatisation de la prise de la Bastille pendant la Révolution : représentations et révisions
}

The Dramatization of the Storming of the Bastille during the Revolution:

Representations and Revisions

\section{Paola Perazzolo}

\section{(2) OpenEdition \\ Journals}

\section{Édition électronique}

URL : https://journals.openedition.org/ahrf/12416

DOI : $10.4000 /$ ahrf. 12416

ISSN : 1952-403X

Éditeur :

Armand Colin, Société des études robespierristes

Édition imprimée

Date de publication : 1 mars 2012

Pagination : 49-68

ISSN : 0003-4436

Référence électronique

Paola Perazzolo, «La dramatisation de la prise de la Bastille pendant la Révolution : représentations et révisions ", Annales historiques de la Révolution française [En ligne], 367 | janvier-mars 2012, mis en ligne le 28 septembre 2012, consulté le 01 juillet 2021. URL : http://journals.openedition.org/ahrf/ 12416 ; DOI : https://doi.org/10.4000/ahrf.12416 


\title{
LA DRAMATISATION DE LA PRISE DE LA BASTILLE PENDANT LA RÉVOLUTION : REPRÉSENTATIONS ET RÉVISIONS
}

Paola PERAZZOLO

\begin{abstract}
Événement-symbole à forte valeur politique, la prise de la Bastille constitue un sujet dramatique très exploité dont la fortune reste moyenne et inconstante. L'analyse de la réception de la production dramatique de l'époque nous éclaire non seulement sur l'histoire du théâtre, mais aussi et surtout sur l'histoire de la Révolution et de l'évolution des mentalités. Malgré les nombreuses réécritures et révisions formelles et/ou idéologiques visant l'adéquation à l'actualité et à un esprit public tout puissant, la fortune des pièces du corpus décroît sensiblement après la deuxième moitié de 1791. Ce déclin, motivé par des raisons multiples, explicite surtout les difficultés et les contradictions consubstantielles à une littérature de circonstance dont l'accueil est soumis aux aléas de l'histoire socio-politique.
\end{abstract}

Mots clés : Bastille, théâtre, spectateurs, critique, dramaturges, écriture de l'histoire

Comme le soulignent Hans-Jürgen Lüsebrink ${ }^{1}$ et Rolf Reichardt ${ }^{2}$ dans leurs travaux de référence, la prise de la Bastille représente à l'époque un événement-symbole à l'immense valeur politique qui fonde la naissance du concept de souveraineté populaire appuyée sur le droit

(1) Hans-Jürgen LÜSEBRINK, «La Prise de la Bastille: archéologie d'un événementsymbole », dans Mélanges de l'École française de Rome. Italie et Méditerranée, t. 104, n. 1, 1992, p. 115-128; id., "Événement dramatique et dramatisation théâtrale. La prise de la Bastille sur les tréteaux français et étrangers », $A H R F, \mathrm{n}^{\circ} 61,1989$, p. 337-355.

(2) Hans-Jürgen LÜSEBRINK et Rolf ReICHARDT, The Bastille. A history of a symbol of despotism and freedom, Duke University Press, (traduit par Norbert Schürer), 1997, p. 38 sq. 
de résistance et qui constitue une rupture dans le champ du discours politique par l'établissement d'un « contre-Panthéon» imaginaire ${ }^{3}$. Son importance symbolique est à la fois fondée et attestée par l'ampleur d'une mise en scène publique qui engendre et perpétue le mythe de la première journée révolutionnaire tout en incitant à l'action patriotique, retraçant ainsi de façon concrète et visuelle la dimension abstraite d'un événement perçu comme intrinsèquement dramatique ${ }^{4}$ et évoqué par de nombreux discours, récits, témoignages ${ }^{5}$, chansons et matériaux iconographiques, ainsi que par de multiples pratiques populaires - les simulacres, le culte des « reliques patriotiques » de l' " Apôtre de la Liberté » Palloy - s'ajoutant aux célébrations officielles.

Il n'est donc pas étonnant que la prise de la Bastille devienne aussitôt un sujet pour les tréteaux de par son importante charge émotionnelle et pathétique, sa valeur politique, didactique et commémorative, sa dimension festive qui permet la participation d'un public de plus en plus élargi et politisé et dont les interventions décident de l'établissement des répertoires officiels, du déroulement des spectacles et de l'apparition de ce que Sophie Marchand qualifie de « répertoire-événement $»^{6}$. La première pièce créée est l'anonyme La Fête du Grenadier ${ }^{7}$, montée au Théâtre de l'Ambigu-Comique le 3 septembre 1789 (50 représentations, dont 22 en $1789,26$ en 1790 et 2 en 1791 $)^{8}$, suivie l'année d'après par Le 14 de juillet de Fabre d'Olivet ${ }^{9}$, créée le 12 juillet 1790 au Théâtre des Délassements Comiques ( 8 représentations en 1790) et reprise « avec des changements analogues aux circonstances » le 21 octobre au Théâtre des Associés

(3) Hans-Jürgen LüSEBRINK, « La Prise de la Bastille : archéologie ... », art. cit, p. 117.

(4) Ibid., p. 337.

(5) Voir Jacques Godechot, La Prise de la Bastille. 14 juillet 1789, Paris, Gallimard, 1989; Lüsebrink et Reichardt aussi signalent les « fifty or so general reports on the storming of the Bastille published in France during 1789, which were supplemented by dozens of leaflets and newspapers items on details », op. cit., p. 47. Cette production est destinée à s'accroître pendant l'anné suivante, voir Hans-Jürgen LÜSEBRINK, « Événement dramatique et... », art. cit., p. 341.

(6) À savoir « un autre type de répertoire, lié à la pratique sociale et à l'investissement politique du spectacle », Sophie Marchand, «Pour une approche culturelle du répertoire révolutionnaire : la lorgnette anecdotique », dans Martial Poirson (dir.), Le théâtre sous la Révolution. Politique du répertoire (1789-1799), Paris, Desjonquères, 2008, p. 217.

(7) La Fête du Grenadier, pantomime nationale et militaire mêlée de chants, de danses, et à spectacle, Paris, Cailleau, 1789. Toutes mes citations sont tirées des éditions ou des manuscrits indiqués, dont je signale la page ou le feuillet entre parenthèses.

(8) Exception faite pour des cas spécifiques, toutes mes informations sur les représentations sont tirées de André Tissier, Les spectacles à Paris pendant la Révolution. Répertoire analytique, chronologique et bibliographique, Genève, Droz, 1992-2002, 2 vols.

(9) Antoine Fabre D’Olivet, Le 14 de juillet 1789, fait historique en un acte et en vers, Paris, Laurens junior, s.d. 
(4 représentations dont 1 en 1790, 2 en 1791 et 1 en 1792) ${ }^{10}$ et par $L a$ Prise de la Bastille, hiérodrame suivi d'un Te Deum de Marc-Antoine Desaugiers ${ }^{11}$ composé à partir de passages de la Bible mis en musique dans la cathédrale de Notre-Dame (13 juillet 1790 et 1791) et dans la salle de l'Opéra, boulevard Saint-Martin, le 25 décembre 1790. En juillet 1790 paraissent aussi deux ouvrages qui ne traitent de la prise du fort que de façon indirecte : La Fête de la Liberté ou le Dîner des patriotes de Charles Ronsin, mise en scène le 12 juillet 1790 au Théâtre du Palais Royal (6 représentations en 1790) puis reprise au Théâtre Français de la rue de Richelieu (2 représentations en juillet 1792) ${ }^{12}$ et La Famille patriote ou la Fête de la Fédération de Collot d'Herbois, une comédie montée le 16 juillet 1790 au Théâtre de Monsieur (17 représentations en 1790) ${ }^{13}$. L'année suivante, on peut compter La Liberté conquise ou le Despotisme renversé de Harny de Guerville $^{14}$, créée le 4 janvier 1791 au Théâtre de la Nation (32 représentations, dont 26 en 1791, 5 en 1792 et 1 en 1793), La Prise de la Bastille de Pierre-Mathieu Parein ${ }^{15}$, La Bastille, ou le Régime intérieur des prisons d'État, drame de J.-J. Thomas (et Guillaume La Forme) ${ }^{16}$ mis en scène le 6 juin 1791 au Théâtre Français Comique et Lyrique (24 représentations en 1791 et 11 en 1792) et La Prise de la Bastille, drame national de JeanJacques Barbot ${ }^{17}$ créé le 25 août 1791 au Théâtre Français de la rue de Richelieu (5 représentations en 1791$)^{18}$.

On peut aussi mentionner d'autres ouvrages qui n'ont jamais été transposés sur les planches : La Prise de la Bastille ou la Liberté conquise

(10) André Tissier précise que, contrairement à l'indication de l'édition, la pièce est créée aux Délassements Comiques, op. cit., p. 173, note 32.

(11) Marc-Antoine Desaugiers, La Prise de la Bastille, Paris, Cailleau, s.d.

(12) Charles-Marie Ronsin, La Fête de la Liberté ou le Dîner des patriotes, comédie en un acte et en vers avec des couplets, Paris, Cussac, 1790.

(13) Collot D’Herbois, La Famille patriote ou la Fête de la Fédération, comédie, Paris, Veuve Duchesne, 1790.

(14) Ce « drame historique » ne nous est transmis que par un manuscrit de souffleur conservé à la Bibliothèque-Musée de la Comédie Française sous la cote Ms. 380 et Mf. 608. Je tiens à remercier $\mathrm{M}^{\mathrm{me}}$ Jacqueline Razgonnikoff de me l'avoir signalé.

(15) Pierre-Mathieu PArein, La Prise de la Bastille, fait historique en trois actes, en prose, et mêlé d'ariettes, Paris, chez Girardin et Madame Lesclapart, 1791.

(16) La pièce n'ayant pas été imprimée, mes informations sont tirées du compte-rendu de la Chronique de Paris du 9 juin 1791 signalé par André Tissier, op. cit., p. 224.

(17) Ainsi que l'indique André Tissier, l'auteur est connu aussi sous le nom de Barbeau, op. cit., p. 232. Je n'ai pas pu retrouver le texte.

(18) Lüsebrink signale aussi La Révolution française, un ouvrage créé le dix août 1792 et constitué par une sorte de «tryptique » dont la prise de la Bastille ne représente que l'un des événements retracés. Voir Hans-Jürgen LüSEBRINK, « Événement dramatique et.... », art. cit., p. 347. 
de Pierre David ${ }^{19}$, Les Imitateurs de Charles IX, ou les Conspirateurs foudroyés de Gabriel Brizard ${ }^{20}$ et Les Deux Prisonniers ou la Fameuse Journée, drame historique et lyrique en trois actes publié en 1792 par Marie-Joseph Martin ${ }^{21}$, dont la singularité mérite qu'on le prenne en considération plus loin. Dans le cas du premier texte, l'absence de mise au répertoire ne s'avère pas étonnante, puisqu'il s'agit d'une pièce médiocre proposée aux comédiens français par un très jeune auteur qui veut exploiter un «sujet [...] presqu'unique » qu' « il est glorieux d'être le premier à $[\ldots]$ traiter » (p. 3). Il est en revanche plus intéressant de souligner la nature hybride de Les Imitateurs de Charles $I X$. Bien plus qu'un texte prioritairement conçu pour les scènes, il s'agit là d'un pamphlet politique écrit sous forme de drame. Plusieurs éléments permettent d'exclure toute possibilité de représentation publique : l'édition semi-clandestine, la présence importante de notes et de commentaires politiques assez véhéments ${ }^{22}$, l'évocation explicite des liaisons amoureuses entre Marie-Antoinette, la duchesse de Polignac et le comte d'Artois - les personnages qui figurent parmi les principaux conspirateurs du complot de juillet 1789, conjuguant de la sorte corruption sexuelle et corruption politique ${ }^{23}$. De plus, comme

(19) Pierre DAVID, La Prise de la Bastille ou la Liberté Conquise, pièce nationale en quatre actes et en prose, s.l., 1790 .

(20) Les Imitateurs de Charles IX, ou les Conspirateurs foudroyés, drame en cinq actes et en prose, par le rédacteur des vêpres siciliennes et du massacre de la $\mathrm{S}$. Barthelemi [Gabriel BRIZARD], Paris, De l'Imprimerie du Clergé et de la Noblesse de France, dans une des caves ignorées des Grands Augustins, 1790.

(21) Marie-Joseph Martin, Les Deux Prisonniers ou la Fameuse Journée, Paris, chezl'auteur et chez Denné, 1792.

(22) À titre d'exemple, je vais citer quelques notes concernant des personnages publics, tels le comte d'Artois ( « On sera peu étonné de voir passer alternativement S.A. de l'expression libertine et du persiflage indécent d'un courtisan sans mœurs ni délicatesse, au langage féroce d'un bourreau du despotisme. Il ne fut jamais agité que par les plus affreuses passions, l'extravagance du jeu, le libertinage et la dissolution, la noire envie, et toute la rage de l'aristocratie », p. 17), la duchesse de Polignac (« La tribade de Polignac peut raisonner en connoisseuse sur ce chapitre; elle a mis en usage tous les goûts, et éprouvé les habitans de tous les pays, même les valets des ambassadeurs Tipo-Sayb, les noirs Africains, \&c. \&c. », p. 18), Berthier (« On n'ignore pas que c'est à cet exécrable intendant que nous avons dû la sécurité que le roi a témoigné sur nos malheurs; l'infâme calomnie empoisonnoit ses rapports. Louis croyoit son peuple heureux. Infortuné monarque! Combien vous êtes trompé! », p. 20), la reine (« La familiarité avec laquelle on s'exprime ici devant la reine, ne paroîtra invraisemblable qu'aux gens dénués de raison. Confondue avec des scélérats, adoptant leurs vues criminelles, les ayant même fait naître, il n'en faut pas davantage pour faire évanouir le rang et la majesté ", p. 24).

(23) En 1869, Eugène Jauffret soulignait déjà la dimension pamphlétaire de l'ouvrage : « Ce fut un déluge de pamphlets sous toutes les formes : dialogues, conversations, drames, comédies, tragédies mêmes. Le premier réquisitoire qu'ils [les auteurs révolutionnaires] lancèrent est intitulé Les Imitateurs de Charles IX, ou les Conspirateurs foudroyés [...] cette pièce, fort curieuse en ce qu'elle exprime la violence des passions politiques, ne fut pas représentée; elle n'aurait pu l'être sans scan- 
l'annonce le titre, l'auteur, un homme de lettres connu pour sa production historique et pamphlétaire et pour avoir édité les Euvres complètes de Rousseau avec Louis-Sébastien Mercier, insiste sur le dévoilement des intrigues politiques qui hantaient l'imaginaire de ses contemporains plus que sur la description du siège ${ }^{24}$, qui fait finalement l'objet d'une ellipse narrative ${ }^{25}$.

Rapidement évacuée dans l'ouvrage de Brizard, la description de l'événement-symbole est aussi escamotée dans deux comédies qui se focalisent sur la glorification de la journée à l'occasion de la Fête de la Fédération - La Famille patriote ou la Fête de la Fédération et La Fête de la Liberté ou le Dîner des Patriotes. Elle est également à peine évoquée dans le dénouement de La Bastille, ou le Régime intérieur des prisons d'État, qui met en scène les souffrances endurées par le prisonnier Dorval jusqu'à ce que les vainqueurs pénètrent dans son cachot lui apportant « la vie \& la liberté ${ }^{26}$. La description de l'action militaire, représentée ou juste rapportée, constitue par contre le climax dramatique des autres pièces du corpus, dont les paratextes traduisent la volonté de référentialité par la négation de tout élément fictionnel : les titres renvoient de façon directe à la célèbre journée ou à l'un de ses traits principaux, les indications génériques explicitent la recherche d'une légitimation historique ou idéologique (voire patriotique) ainsi que le choix majoritaire de ces genres nouveaux - le fait historique, la pantomime nationale et militaire, le drame patriotique, historique ou national - qui se détachent de la dramaturgie classique pour se fixer plutôt, ainsi que le rappelle Pierre Frantz, « aux frontières mêmes du théâtre, de l'opéra, de la fête, de l'art oratoire, dans des expériences inédites du regard, des sensations et des émotions d'une force et d'une nouveauté telles qu'on ne pouvait les oublier $»^{27}$.

dale et sans trouble », Eugène JAUFFRET, Le théâtre révolutionnaire (1788-1799), Paris, 1869, Genève, Slatkine Reprints, 1970, p. 92 et p. 95.

(24) La « Table des personnages » s'avère déjà explicite à cet égard : il n'est question que d'« un électeur », du célèbre « Grenadier aux Gardes Françaises » et d'une «Troupe de Gardes et de Citoyens », alors que la liste des personnalités publiques est bien plus nourrie : y figurent le roi et la reine, le comte d'Artois, les princes de Condé et de Conti, la duchesse de Polignac, le duc d'Orléans et de Bourbon, le duc du Chastelet, le Prince de Lambesc, le duc de Noailles, le comte de Guiche, l'Archevêque de Paris, Bailly, La Fayette, le marquis de Launay, Foulon, Berthier et Flesselles (p. 7).

(25) Une note précise : « Je saute sur l'événement de la prise ou plutôt du miracle de la Bastille : les relations qui en ont été données au public suffisent pour son instruction. Ce prodige glaça le sang des aristocrates, principalement celui de l'illustre et méprisable chef de parti » (p. 66-67).

(26) Voir Chronique de Paris, 9 juin 1791.

(27) Pierre Frantz, «Les tréteaux de la Révolution (1789-1815)», dans Jacqueline de Jomaron (dir.), Le Théâtre en France, Paris, Armand Colin, t. II, 1989, p. 24. 
Encore, dans la plupart de leurs avis, lettres ou préfaces, les auteurs réaffirment leur volonté de sacrifier tout élément fictionnel et dramatique sur l'autel de la véracité historique ${ }^{28}$ afin de «mettre sous les yeux des Français ce qu'ils ont fait de plus glorieux $\&$ de plus heureux $\&$ de leur répéter, comme dans un miroir, le tableau qu'eux-mêmes ont composé », pour reprendre les mots de David ${ }^{29}$.

La lecture de ces ouvrages confirme la volonté de subordonner le rôle du dramaturge à celui de l'historien : ainsi que l'a rappelé la critique, la chronologie des événements suit de près celle retracée dans les sources à disposition ${ }^{30}$; la topographie évoque les lieux principaux de l'action (la Bastille et la place de 1'Hôtel de Ville) ${ }^{31}$; l'insertion d'aphorismes révolutionnaires, d'expressions reprenant l'iconographie contemporaine, de formules tirées des journaux ou des discours politiques ${ }^{32}$ témoigne bien de la porosité des genres, typique de l'époque, comme de la tentative d'effacer toute frontière entre fiction et événement représenté grâce à une « écriture-slogan $»^{33}$ qui a pour conséquence première le délaissement de la cohérence dramaturgique ${ }^{34}$ et de la valeur littéraire, ainsi qu'en témoigne le compte-rendu de La Liberté conquise des Affiches, Annonces et Avis divers du 6 janvier 1791 :

«L'Auteur, en compulsant le Journal des Débats sans doute, \& tout ce qui a été écrit sur la Révolution, a inséré dans la Pièce des fragments de discours \& des principes que l'impression nous reproduit tous les jours sous mille formes, ce qui donne souvent de l'élévation à son style [...] $L a$ Liberté conquise [...] doit son intérêt à la véracité \& au rapprochement des faits : si l'on n'y reconnoît pas un grand mérite littéraire, on y trouve au moins un très-grand Patriotisme, \& l'un est maintenant plus sûr du succès que l'autre. Cet Ouvrage a été reçu avec l'enthousiasme d'un Peuple qui est dévoré de la soif de la Liberté ».

(28) Voir Hans-Jürgen LÜSEBRINK, «Événement dramatique et ... », art. cit., p. 353 sq.

(29) Pierre DAVID, op. cit., p. 2, je souligne.

(30) Voir Hans-Jürgen LÜSEBRINK, « Événement dramatique et... », art. cit., p. 344 sq.

(31) Voir Philippe CoRno, « La Prise de la Bastille sur la scène révolutionnaire : un théâtre à la limite », dans Martial Porrson (dir.), Le théâtre sous la Révolution...., op. cit., p. 329.

(32) Voir Hans-Jürgen LüSEBRINK, «Événement dramatique et ... », art. cit., p. 351.

(33) Sophie Marchand, art. cit., p. 217.

(34) Les auteurs paraissent en avoir parfois conscience : David écrit en note que « Pour éviter le refroidissement de l'action, on pourrait supprimer à la représentation tout ce qui se trouve marqué par des guillemets » (p. 53), à savoir une longue tirade du premier citoyen expliquant à un prisonnier les événements patriotiques des deux derniers jours. 
Au niveau scénique, la volonté d'authentification s'incarne dans un effort remarquable de réification de l'événement afin de rendre des " "effets de réel" spécifiques ». À savoir, pour reprendre l'expression de Lüsebrink, « le simulacre de la prise de la Bastille, le fracas de l'action militaire, la présence sur scène d'objets authentiques provenant de la Bastille $»^{35}$. Sur les tréteaux des grands théâtres - c'est le cas de La Liberté conquise au Théâtre de la Nation ou de La Prise de la Bastille dans la salle rivale de la rue de Richelieu - l'effort scénique peut se concrétiser dans des décors hyperréalistes ${ }^{36}$, des costumes très variés et une distribution nombreuse dont le but est de retracer toutes les actions et tous les personnages et de restituer la puissance du peuple, hétérogène dans sa composition mais uni dans le combat patriotique ${ }^{37}$.

A priori voués au succès de par leur valeur politico-patriotique et leur charge pathétique et émotionnelle, beaucoup d'ouvrages jouissent en fait d'une faveur mitigée, ainsi que le rappelle Lüsebrink quand il évoque les faiblesses esthétiques et stylistiques de la plupart des textes et les difficultés de leur mise en scène ${ }^{38}$. À ce propos, l'on peut ajouter d'autres considérations. D'après le répertoire d'André Tissier, La Fête $d u$ Grenadier ne jouit que de deux représentations après 1790, Le 14 de juillet est très peu repris en 1791 et la pièce de Collot point du tout. En ce qui concerne les créations de 1791, La Liberté conquise rencontre une faveur impressionnante ${ }^{39}$ pendant la première partie de l'année mais

(35) Hans-Jürgen LÜSEBRINK, «Événement dramatique et ... », art. cit., p. 355.

(36) Le décorateur Lesueur avait reconstruit « toute la Bastille sur plusieurs plans » : tout au début, c'est la forteresse « de face », suivie par le pont-levis, ses bascules, la grand tour du fond et son intérieur sur deux plans, les appartements du gouverneur, des arbres, l'arsenal et par l'évocation des cuisines du fort et des boutiques, des maisons - complètes de fenêtres réelles, de volets et des fermetures peintes des deux côtés - devant la Bastille; deux autres châssis offrent enfin la vue de toute la ville. Lesueur prévoit aussi les canots pour les lampions et la présence de deux pièces de canons sur scène. Voir Dossier ARD Lesueur, Mémoire des Décorations, Archives de la Comédie Française.

(37) Le journaliste du Courrier des 83 départements du 25 août 1791 signale que La Prise de la Bastille est « précisément le tableau extrait de l'événement du 14 juillet », et « trop exact peutêtre » puisque rien n'est exclu : « L'expédition du peuple au Garde-Meuble, que l'on a rapprochée de celle faite à l'Hôtel des Invalides; la réunion de plusieurs soldats des divers régimens, qui sont venus se ranger sous l'étendard de la patrie. Ce qui n'est pas exempt de défauts, puisque la distribution est trop nombreuse, au point que la pièce paraît ne pas pouvoir « comporter ce fouillis [...] d'hommes de toute classe, de tous états » et « une foule d'hommes de toute espèce, dont les différens costumes \& le mouvement fatiguoient la vue $\&$ genaient la marche de l'action ».

(38) Hans-Jürgen LÜSEBRINK, « Événement dramatique et ... », art. cit., p. 346.

(39) Parmi les nombreux comptes-rendus qui relatent le succès du spectacle, voir Affiches, Annonces et Avis divers, 6 janvier 1791, Mercure de France, 22 janvier 1791 et Journal de Paris, 5 janvier 1791. 
apparaît peu à l'affiche par la suite ${ }^{40}$, alors que d'autres pièces trouvent peu de place sur les planches : refusée dans la capitale, La Prise de la Bastille de Parein n'est représentée qu'en province avec un piètre succès ${ }^{41}$; demandée par le parterre, qui réclame un spectacle qui puisse concurrencer le succès de La Liberté conquise au rival Théâtre de la Nation, La Prise de la Bastille de Barbot n'est jouée que cinq fois. Certes, au point de vue dramatique et scénique l'ouvrage reste bien inférieur à celui d'Harny, caractérisé par des « spectacular theatrical effects $[\ldots]$ that let the contemporary audience sensually experience the heroic deeds of the conquerors $»^{42}$ ainsi que par " un nouveau langage politique de la rue et l'identification au nouvel ethos du citoyen-militaire $»^{43}$. Les comptes-rendus signalent les défauts de l'ouvrage de Barbot tout autant qu'un changement des mentalités : Le Courrier des 83 départements du 25 août loue les décors, le jeu des acteurs, le style et le patriotisme de l'auteur, mais critique l'excessif souci d'authenticité qui rend le texte embrouillé, confus ${ }^{44}$ et finalement assimilable à une fiction ( « on croyoit assister à une tragédie de Sapeskear [sic] »). De même, le journaliste de la Chronique de Paris du 27 août évoque le bon succès de la première, loue le jeu des acteurs et les costumes, mais glisse sur l'exposition d'un sujet déjà connu ${ }^{45}$ pour exprimer une certaine perplexité quant à la lon-

(40) D'après les Affiches, Annonces et Avis divers, le drame, monté le 26 et 27 juin 1791, ne sera plus repris jusqu'à octobre de l'année suivante, et ne sera joué qu'une fois "par et pour le peuple » en août 1793 .

(41) Refusée par le Théâtre des Italiens, la pièce ne figure pas dans le répertoire de Tissier; Eugène Jauffret atteste pourtant sa présence sur les planches quand il écrit que « sa pièce [de Parein] fut jouée. Le fut-elle par l'effet de l'action judiciaire [l'auteur avait eu recours au tribunal contre les comédiens, sans gagner sa cause] ou par celui des événements politiques, dont l'influence emportait l'opinion d'un mouvement de plus en plus rapide? Quoi qu'il en soit, Pierre-Mathieu Parein eut l'avantage d'entretenir quelque temps le public de ses griefs et de sa gloire », op. cit., p. 123. Pour son compte, Lüsebrink précise que « Parein's drama was a complete flop. It was rejected by the Theâtre des Italiens and then received without enthusiasm in the few performances it experienced (for exemple, in Rouen in 1795) », A history..., op. cit., p. 99.

(42) Hans-Jürgen LÜSEBRINK, ibid.

(43) Id., «Événement dramatique et... », art. cit., p. 354.

(44) «L'auteur paroît avoir oublié dans la représentation ce vers de Boileau : Le vrai peut quelquefois n'être pas vraisemblable [...] enfin, les préparatifs du siège, \& cette catastrophe même qui termine la pièce : tous ces événements, dis-je, ont été retracés avec une fidelité étonnante, \& sans d'autre confusion que celle qu'a cru devoir y mettre l'auteur, qui a tout sacrifié à la vérité, qui est devenue effrayante, surtout au moment du siège. L'incendie du gouvernement, la trahison de Delaunay, la fusillade qui a engagé l'action, le combat qui l'a suivie, tout, jusqu'aux prisonniers arrachés des cachots, a été representé d'après nature; enfin, aucune des circonstances n'a été oubliée (On croyoit assister à une tragédie de Sapeskear [sic]».

(45) « C'est une représentation fidèle \& dialoguée de la fameuse journée du 14 juillet. Ainsi nous n'en ferons pas l'analyse ». 
gévité d'un spectacle monté presque exclusivement à la demande du parterre :

« Cet ouvrage avoit été demandé par le parterre; il a réussi, \& les entrepreneurs seront dédommagés des frais que son exécution nécessite; mais il pourroit se faire qu'il tombât, \& que cette violence leur fût très nuisible. Dans le tems du régime prohibitif, on avoit sans doute le droit de forcer les comédiens à jouer les pièces qui ne pouvoient être représentées ailleurs ; mais aujourd'hui [...] c'est une vexation manifeste de contraindre leurs volontés ».

Dans d'autres comptes-rendus se faufilent des critiques encore plus intéressantes qui témoignent d'une possible saturation du public à l'égard d'un sujet désormais peu actuel et beaucoup exploité : le journaliste de La Feuille du jour du 30 août reste assez laconique - « Cet ouvrage est écrit comme tous ceux que le patriotisme a dictés $»-$, alors que dans son Almanach des spectacles Beffroy se montre plus tranchant à l'égard de ce qu'il définit comme une « platitude, dont le sujet est si rebattu et a été beaucoup mieux traité partout ailleurs ». D'après lui, l'auteur de ce « drame patriotico-politico-Jacobite » aurait en vain cherché le soutien des Jacobins, priés par une circulaire de venir applaudir la pièce, puisque «le Public, qui n'avait pas reçu de circulaire, a sifflé ce qui était mauvais; et il est peu de choses dans la Pièce qui n'ait été sifflé ». Non seulement l'ouvrage serait moyen, mais la représentation de l'événement serait désormais peu originale, ainsi que l'affirme Beffroy : "C'était bien-là le cas de représenter encore des Bastilles, après deux ans et plus, pendant lesquels toutes les oreilles Parisiennes avaient été tant embastillées ${ }^{46}$.

La diminution de l'intérêt du public à l'égard de la représentation de l'événement fondateur de la Révolution pourrait en effet intéresser d'autres ouvrages du corpus : La Bastille, ou le Régime intérieur des prisons-d'État semble aussi payer le déclin de la vogue d'un sujet que l'auteur essaie pourtant de varier, insistant sur la rhétorique du pathétique plus que sur l'action militaire et l'esthétique du simulacre. La peinture détaillée des horreurs et des injustices perpétrées par les tyrans - le ministre et le gouverneur - contre les victimes du despotisme fournissent une action fort louée dans la Chronique de Paris et dans l'Alma-

(46) Louis-Abel BefFroy de ReIgny, Almanach général de tous les spectacles de Paris et des provinces pour l'année 1791-1792, Paris, Froullé, 1791-1792, p. 239. 
nach, Beffroy ne déplorant cette fois que le manque d'opportunité de la création : "La Bastille est très-supérieure à tout ce qu'on a fait dans d'autres Spectacles sur le même sujet. Scènes rapides, bien enchaînées ; tableaux vrais, attachants; style correct; idées vraies et fortes; contrastes heureux et bien amenés, dénouement à-la-fois naturel et frappant. S'il eût été joué un an plus tôt, il eût obtenu un succès brillant, durable et mérité $\gg^{47}$.

Après deux ans d'« embastillement », la réception moyenne des pièces du corpus pourrait s'expliquer par des raisons multiples, plus ou moins pratiques - l'importance des frais que peuvent comporter ces représentations-commémorations, la présence de deux pièces qui ont déjà rencontré la faveur des spectateurs, la rapidité de l'évolution des goûts du public, la diminution de son enthousiasme à l'égard d'un sujet de moins en moins actuel et que la surexposition médiatique rend fort exploité - qui viennent s'ajouter à celles déjà évoquées par Lüsebrink. Il resterait peut-être à prendre en considération un autre aspect qui explicite les contradictions inhérentes à cette littérature de circonstance à la nature fortement politique et dont l'accueil est plus que jamais soumis aux aléas de l'histoire et de l'évolution des mentalités. Ainsi que le rappelait Philippe Corno, la tentative de restitution authentique de l'événement implique forcément l'adhésion à une position idéologique et la formulation d'une interprétation politique sur la première journée révolutionnaire, notamment en ce qui concerne la représentation de la brutalité suivant la prise $\mathrm{du}$ fort et de la participation du monarque au complot aristocratique évoqué dans quasiment tous les ouvrages ${ }^{48}$.

À ce propos, il est à considérer que dans les premières pièces la violence populaire est stigmatisée mais finalement légitimée parce que consubstantielle à l'insurrection politique et à la prise de conscience de la naissance d'une puissance nouvelle - la Nation. D'un côté, il s'agit de répondre à l'arbitraire de l'Ancien Régime en faisant coïncider vengeance et justice populaire; de l'autre, de frapper sans hésiter tout ennemi en sacrifiant l'humanité sur l'autel des valeurs patriotiques. Comme le souligne Jacques Godechot, on voit que la justification de la brutalité pose dès sa naissance la Révolution sous le double signe des idéaux philosophiques et des massacres considérés comme une nécessaire réponse au despotisme et aux intrigues des privilégiés ${ }^{49}$. Hantés par l'obsession d'un

(47) Ibid., p. 195.

(48) Voir Philippe CoRno, art. cit., p. 334.

(49) Jacques Godechot, op. cit., p. 377. 
complot aristocratique que Mirabeau décrit comme «l'avant-scène de la Saint-Barthélemy $\gg^{50}$, les discours contemporains ont tendance à acquitter les violences, ainsi que le montrent quelques récits ${ }^{51}$, des chansons de l'époque ${ }^{52}$, des témoignages célèbres comme la lettre de Babeuf concernant le « trop juste ressentiment » du peuple ${ }^{53}$ ou encore les propos avancés par Loustalot dans Les Révolutions de Paris ${ }^{54}$. De ce point de vue, les pièces ont encore tendance à se poser en miroir du sentiment collectif. Bien que rapidement sublimée dans la dimension festive, dans les premiers ouvrages la sauvagerie populaire est évoquée et légitimée : dans La Fête du Grenadier, le peuple avide de nouvelles de l'action militaire apprend que « Le fort est pris; sous nos coups ils expirent / Des trahisons

(50) Ibid., p. 291.

(51) Dusaulx retrace les violences (« Ils nous conjuraient de les venger [...] leurs prières ressemblaient à des ordres, et plus d'une fois il n'a pas été possible d'y résister [...] le peuple [...] moins touché du sort des prisonniers que l'on trainait, que l'on immoloit de temps en temps, que de ce grand spectacle [la foule en fête] voyoit couler le sang avec stupeur. Se dissimulant le carnage, il applaudissoit aux dépouilles singulières promenées sous ses yeux ») et évoque une foule en proie à un délire de vengeance dont l'auteur excuse de la sorte la brutalité : " dans le tumulte et la fermentation de tous ces courageux égarés par l'impetuosité des premiers mouvements, quelques victimes furent immolées; mais toutes ne furent pas également regrettables : ne l'imputons qu'à l'aveugle fatalité. Notre nation, violente quelquefois, surtout quand elle est excitée, ne fut jamais cruelle ni atroce de sangfroid ». De l'Insurrection parisienne et de la prise de la Bastille, Paris, Debure, 1790, p. 116 et p. 132.

(52) À titre d'exemple, citons la Prise du gouverneur, sa fin tragique ainsi que celle de Foulon, Flesselles, Bertier, composée en 1790 : «Le gouverneur perfide/Veut en vain s'échapper, / Un soldat intrépide / Parvint à le happer. (Refrain) Eh mais oui-dà! / Comment peut-on trouver du mal à ça? / Oh! Nenni-dà ! / Comment peut-on trouver du mal à ça ! / Ennemi de la France, / Votre règne est passé; / Le temps de la vengeance / Est enfin arrivé (Refrain.) À de Launay, Flesselles, / À Bertier et Foullon, / On met une ficelle / Au-dessous du menton. (Refrain) », Michel Delon et Paul-Édouard Levayer (eds.), Chansonnier Révolutionnaire, Paris, Gallimard, 1989, p. 60 .

(53) « À mon arrivée à Paris on ne s'entretenait que d'une conspiration dont M. le comte d'Artois et d'autres princes étaient les chefs. Il ne s'agissait rien moins, pour eux, que de faire exterminer une grande partie de la population parisienne et de réduire ensuite à la condition d'esclave tout ce qui, dans la France entière, n'aurait échappé au massacre qu'en se mettant humblement à la disposition des nobles, en tendant sans murmurer les mains aux fers préparés par les tyrans. Si Paris n'eut pas découvert à temps cet affreux complot, c'en était fait, jamais crime plus épouvantable n'aurait été consommé. Ainsi n'a-t-on pu songer qu'à tirer une éclatante vengeance de cette perfidie dont il n'y a pas d'exemple dans l'Histoire. On s'y est résolu, et on n'épargnera ni les auteurs principaux de la conjuration ni leurs adhérents. Les exécutions ont commencé sans épuiser un trop juste ressentiment. La fureur du peuple est loin d'être apaisée par la mort du gouverneur de la Bastille », dans Jacques GODECHOT, op. cit., p. 375.

(54) « Français, vous exterminez les tyrans! Votre haine est révoltante ! Elle est affreuse [...] Mais vous serez libres enfin! je sens, ô mes concitoyens! combien ces scènes révoltantes affligent votre âme; comme vous, j'en suis pénétré. Mais songez combien il est ignominieux de vivre et d'être esclave! », dans ibid., p. 376. 
ceux qui sont l'instrument » (p. 6) dans la pièce de David ${ }^{55}$ et de Fabre $\mathrm{d}^{\prime} O \mathrm{Olive}^{56}$ le grenadier justifie les épisodes de violence au nom d'une vengeance teintée de justice, et l'on peut bien imaginer que Brizard saisit l'occasion pour rappeler la légitimité des représailles populaires à l'égard du marquis de Launay ${ }^{57}$ ou de Jacques de Flesselles, explicitement accusé de trahison $^{58}$.

Les ouvrages suivants restent en revanche plus elliptiques à ce propos, en accord peut-être avec un discours politique officiel qui, à partir de 1790, essaie d'effectuer un refoulement des figures individuelles et populaires et de domestiquer la dimension insurrectionnelle de l'événement et le droit à la résistance violente pour mettre l'accent sur l'unité nationale et la portée collective de la journée ${ }^{59}$. Parmi les ouvrages parus en 1791,

(55) «Courageux citoyens, \& vous mes camarades, vous êtes suffisamment vengés. Nous avons été témoins du supplice que le gouverneur \& ses complices viennent de souffrir; il ne pouvait être plus rigoureux : ils sont morts. [...] ma haine \& ma juste fureur se sont évanouies à la vue de leur trépas, car enfin c'étaient des hommes [...] nous les avons conduits jusques sur la place de l'hôtel de ville, \& là un peuple immense, furieux \& impatient de se venger, nous a arraché l'inhumain gouverneur, l'a foulé aux pieds, l'a percé de mille coups, \& enfin lui a tranché la tête, qu'on a mise aussitôt au bout d'une lance, $\&$ dont on rassasie les regards avides de tout le peuple. Tâchons, Messieurs, d'arrêter le cours de cette dernière vengeance; elle est barbare, révoltante : elle est indigne des Français. D'ailleurs, les crimes des traîtres sont expiés » (p. 57); la mort de Flesselles est par contre mise sur le compte de traîtres inconnus craignant qu'il ne dévoile d'autres complices et qui « se sont saisis avec violence du prévôt des marchands pour lui brûler la cervelle, \& sa tête est portée auprès de celle du zélée partisan de ses crimes » (p. 58).

(56) « Sans doute vous savez quel fut son triste sort? / Entraîné par un peuple avide de sa mort. / Il en fut déchiré. Cet horrible supplice / Fut trop prompt, trop cruel, pour être une injustice; / il méritait la mort. Mais ces barbares coups, / S'ils sont dignes de lui, sont indignes de nous » (p. 48).

(57) Au début du troisième acte, l'auteur rappelle que « Cet acte occupe la journée du mardi 14 juillet, et la nuit de ce même jour au mercredi 15. Quoique tous les papiers publics en ayent fourni les détails, on ne sera, je crois, pas faché de suivre cette atrocité dans tous ses points ». Si Brizard évoque le " détail affligeant, et bien fait pour nous faire gémir éternellement sur la barbarie des grands » (p. 80), il montre aussi les vaincus traînés à l'hôtel de ville au cri de « Les monstres! Les barbares!» (p. 79) et traités de «criminels de leze-nation» (p. 83), ainsi que la description des citoyens criant « au reverbère! au reverbère! » ou " Point de grace, point de grace. », de la sorte que « Tout le peuple emploie la violence, pour descendre au supplice les sieurs de Launay et du Pujet» (p. 81). De même, une note décrit « le peuple [qui] s'occupe à déplacer le reverbere du coin du roi. Il y suspend une corde destinée à messieur de Launay et du Pujet, et à quelques invalides : de tems en tems il s'interrompt, en s'écriant, faites-les descendre! » (p. 80).

(58) L'accusation de Flesselles est renforcée par une note en bas de page : «A-t-on vu jamais l'exemple d'un pareil artifice? Aristocrate infame, tu couvres ta perfidie d'un voile encore plus atroce, que tu parois désirer le bonheur d'un peuple qui t'a confié sa destinée. Dis-moi, infernal bourreau du despotisme, est-ce le moment de refléchir, quand le glaive est suspendu sur notre tete, et que l'on ne nous laisse d'autre choix qu'un affreux esclavage, ou la mort?» (p. 50).

(59) Hans-Jürgen Lüsebrink, « La Prise de la Bastille : archéologie... », art. cit., p. 123; Lüsebrink fait encore remarquer que cette tendance sous-tend bien les discours de 1793-1794: « While the military side of 14 July [...] takes up more and more room in speeches on 14 July, other 
l'élément fictionnel de La Liberté conquise (dont l'action se passe dans une ville de frontière qu'on resitue aisément à Paris) permet à Harny de terminer sa pièce sur la dimension festive, sans aborder ni la question délicate des massacres ni celle de la participation royale au complot. On peut de même avancer l'hypothèse que le texte de Thomas, centré sur le régime intérieur du fort plus que sur sa prise, fait aussi l'économie du récit des violences, alors qu'il est intéressant d'examiner la position de Parein, qui fonde la validité de son ouvrage sur l'authenticité de sa restitution d'un événement auquel il a participé. Sa proclamation de fidélité à l'histoire l'empêche évidemment de passer sous silence des faits récents et encore bien ancrés dans la mémoire collective, et l'auteur se contente donc d'éluder toute évocation directe, rapportant que le Gouverneur est entraîné « à l'hôtel de ville devant les électeurs, pour lui faire rendre compte de son infâme trahison » et qu' " il disparaît avec les cinq citoyens qui sont entrés les premiers dans le fort $\gg$ (p. 59).

Il faut encore souligner que le désir populaire de vengeance contre l'arbitraire de l'Ancien Régime ne se concrétise pas, du moins pendant un premier temps, par la mise en accusation directe de Louis XVI, encore considéré comme le père bienfaisant de la patrie. Le souverain a certes été trop naïf et peu attentif au bonheur de sa nation, mais il a été égaré par ses mauvais conseillers - notamment ses frères et la reine - qui l'ont empêché de connaître la situation réelle de son peuple. Les premiers ouvrages du corpus réaffirment en général l'innocence du monarque et/ou sa bienfaisance ${ }^{60}$, ce qui témoigne d'une interprétation idéologiquement modérée et de la conception d'une Révolution ayant pour but l'établissement d'une monarchie constitutionnelle. C'est là une position de plus en plus difficile

elements are consciously or unconsciously pushed into the backgrounds and repressed : the fear of a conspiracy, the scene of massacre, the descriptions of violence, but also the triumph of the conquerors of the Bastille and the portrayal of individual Vainqueurs », A history..., op. cit., p. 159.

(60) La Fête du Grenadier se termine par la célébration de la Ville, de la Liberté et du Roi, dont l'on fait défiler un buste avec l'inscription "Au Restaurateur de la Liberté » pendant qu'on chante ces couplets : « Français, voilà votre Monarque, / De tous les Rois le plus chéri! / Dans son cœur il porte la marque / Qui fait encore bénir Henri. / Bonté, bienfaisance, / Voilà ses vertus \& sa loi! / C'est librement qu'on peut crier en France : / Vive le Roi! Vive le Roi! (bis) » (p. 13); Collot d'Herbois évoque Louis XVI comme « le Roi, ce bon Roi [...] au milieux d'eux [les membres de l'Assemblée Nationale] comme un père parmi ses enfants, qui sembloit dire d'avance $\&$ au fond du cœur, ce qu'un moment après il a juré : je maintiendrai la Liberté » (p. 40); Brizard aussi a soin pendant tout son ouvrage de souligner que le souverain est tout à fait étranger au complot, alors que seul David semble soulever quelques doutes à cet égard : au major qui, instruit du complot, demande si « un roi que la bonté caractérise a prononcé un ordre aussi cruel [tirer sur les parisiens] » le capitaine répond : «Vouloir approfondir de semblables secrets est un crime; ne nous en rendons point coupables. Notre soumission est aveugle : le roi commande, nous devons obéir » (p. 10-11). 
à tenir pendant les années suivantes, quand l'attitude ambiguë et antipatriotique de Louis XVI, et surtout la tentative de fuite de juin 1791, minent irrévocablement l'image du « bon roi Louis ». La réception des pièces du corpus se fondant sur leur capacité d'adhérer à la circonstance et à l'esprit public, il est impossible que les changements socio-historiques en question ne posent pas de problème puisqu'ils rendent désuète l'interprétation idéologique de la plupart des ouvrages. L'on assiste donc parfois à la mise en place d'une opération de révision qui trouve son expression concrète dans la réécriture des textes ou, pour les nouvelles pièces, dans un effort de neutralité idéologique.

La pièce d'Harny, créée en janvier dans une salle peu susceptible de passer pour un foyer révolutionnaire ${ }^{61}$, constitue un exemple intéressant de réécriture. Alors qu'en début d'année les patriotes célèbrent la prise de la forteresse au cri de « Vive le Roi! Vive la Nation! », le 26 juin - premier jour de réouverture des salles après Varennes - la pièce figure sur une affiche qui ne comporte pas la mention traditionnelle de « Comédiens ordinaires du Roi », ce qui signale un changement laissant supposer que le texte aussi commence à subir, tout comme l'affiche, une révision au niveau du style et des contenus. Le manuscrit présente, de la main de Delaporte (le secrétaire-souffleur de la troupe), de nombreuses variantes de suppression ou de modification qui signalent l'adéquation du texte à l'actualité par rapport à des concepts fondant la res publica: la structure hiérarchique de la société, l'idée de souveraineté absolue, le bien-fondé des prétentions des Grands, la figure de Louis XVI. Toutes les allusions à la gloire et à la bienfaisance du monarque sont raturées et/ou remplacées. Les variantes, espacées dans le temps et souvent difficiles à dater, peuvent être quantitativement peu importantes et porter sur quelques mots ou phrases ${ }^{62}$, ou bien concerner des portions de texte plus grandes, comme c'est le cas à la fin du quatrième acte, lorsque les patriotes s'apprêtent à formuler le serment rituel avant d'attaquer la forteresse. Si à l'origine le

(61) D’après les Registres des feux n. 130.21 des Archives de la Comédie Française, tous les Noirs les plus célèbres jouent un rôle lors de la première, ce qui ferait penser que la pièce avait été soumise à la partie modérée de la troupe, voir aussi Noëlle GuiBERT-Jacqueline RAZGONNIKOFF, Le Journal de la Comédie française. 1789-1799. La comédie aux trois couleurs, Sides, Antony, 1989, p. 130.

(62) Telles, à titre d'exemples, les expressions «>Bénir le Roi bienfaisant qui a préparé le bonheur de son peuple $<$ » (f. 18), «>la gloire du trône $<$ » (f. 47), «>les vertus d'un roi citoyen $<$ » (f. 58), « des citoyens égaux qui concouraient $>$ avec le monarque $<$ à la formation des lois » ou le «>Vive le roi $!<»$ à la fin de l'ouvrage. Pour une plus grande clarté, j'indique les ratures entre > $<$ et les ajouts entre ${ }^{\wedge} \wedge$. 
maire Verneuil jure fidélité à la « nation », à la « loi » et au « roi », toute mention à la monarchie est par la suite effacée - «C'est à la face de l'univers, en présence de l'Être suprême que je jure d'être fidèle à la nation, à la loi et au roi » (f. 113, liasse) -, alors qu'un premier becquet ${ }^{63}$ superposé ne comporte aucune allusion à la figure du monarque et qu'un deuxième affiche des propos véhéments que les tons adoptés et l'allusion à la République « une et indivisible » permettent de dater après septembre 1792 :

«Voici l'instant de venger l'humanité trop longtemps outragée, et de reprendre un pouvoir que les tyrans ont usurpé sur les peuples. Le plus grand ennemi des hommes, c'est un roi. Jurons de n'en souffrir plus en France. Que le titre de roi, que ce titre odieux ne souille plus le sol heureux de notre patrie. Que les images des tyrans, livrées aux flammes et dispersées par les vents annoncent à l'univers que la nation française est vraiment libre! Proscrivons jusqu'au souvenir de la royauté [...] unis dès cet instant par un pacte indissoluble, formons une République une et indivisible; et jurons tous que nous voulons la République ou la mort [...] c'est à la face de l'univers, en présence de l'Être suprême, que je jure de maintenir la liberté et l'égalité, de défendre la République et de la défendre jusqu'au dernier soupir » (f. 113-114, deuxième becquet).

Tout comme La Liberté conquise, d'autres pièces subissent une opération de révision. L'édition de La Fête de la Liberté ou le Dîner des patriotes présente des corrections manuscrites apportées à l'occasion des deux reprises de juillet $1792^{64}$, et ces variantes concernent souvent la suppression de vers mentionnant le "bon roi Louis ${ }^{65}$. Il en va de même de Le 14 de juillet de Fabre d'Olivet, dont le texte publié diverge sensiblement par rapport à deux manuscrits autographes ${ }^{66}$ qui font état de nombreuses corrections sur liasse et sur becquet. L'un d'eux, proba-

(63) Becquet : modification que l'auteur d'une pièce apporte à son texte lors d'une répétition.

(64) La page de titre présente l'ajout manuscrit selon lequel la pièce a été « remise le 15 juillet $1792 »$

(65) «Qui rend >du bon Louis tous les sujets>^les citoyens^ égaux » (p. 6); «Ah! çà, n'oublions pas de boire à la santé $/>$ Du bon roi des Français... Car c'est aussi sa fête. / A lui. / $<\wedge$ de la Nation; c'est aujourd'hui sa fête` (Tous les convives ensemble). $>$ Vive le Roi $!\langle\wedge$ Vive la Nation !^ » (p. 36); «>[Le duc] J'ai pris mon cœur pour guide, et mon roi pour modèle. / Au bonheur de son peuple il s'est sacrifié : / il s'en est bien déclaré le père : / Avec tous ses enfants, je veux agir en frère $<$ » (p. 38); «>Et chantons, vive la loi /La nation, et le roi<» (p. 42); «L'armée entière s'écrie/ $>$ Avec le peuple et le roi<^${ }^{\wedge}$ Des tyrans soyons l'effroi !^ / Vaincre ou mourir pour la loi » (p. 43).

(66) Voir BN Ms., Fr. 9254, signalé dans André Tissier, op. cit., p. 473. Les deux manuscrits, écrits de la même main, sont indiqués comme autographes dans le catalogue. 
blement utilisé pour la reprise d'octobre, porte la mention «avec des changements analogues aux circonstances (aut.) » sur la page de titre ${ }^{67}$, ce qui permet de supposer que les variantes sur becquet, sans pouvoir être datées, sont intervenues après octobre 1790. Parmi d'autres corrections purement stylistiques ou concernant différents épisodes, certaines visent la suppression de la célébration de la gloire et des bienfaits du monarque ${ }^{68}$ ou l'abrogation de toute proclamation d'innocence de celuici :

«>Vous [les soldats] verrez que vos mains, fatalement trompées, / Du sang de vos amis allaient être trempées; / Qu'un roi, dont l'univers proclâme les bienfaits, / N'a pas dit aux sujets d'égorger les sujets; / N'a pas armé le fils contre son propre père; / $\mathrm{Au}$ frère n'a pas dit d'assassiner son frère: /Qu'il est bon, qu'il est juste, \& que, sans le trahir, / À des ordres surpris on peut désobéir <» (p. 13 de l'édition, f. 204 r et f. 226 v.

Fortement investis par le politique et par la nécessité d'une difficile adéquation à un esprit public aussi puissant que soumis à des changements rapides, ces ouvrages composés dans l'urgence de la commémoration véhiculent une position idéologique de plus en plus difficile à tenir. Alors que La Bastille, ou le Régime intérieur des prisons-d'État se concentre sur la description des abus du despotisme de l'intérieur de la forteresse plus que sur des questions politiquement plus délicates - d'où probablement sa présence à l'affiche aussi en 1792 -, la pièce de Parein, publiée en juillet 1791, enregistre déjà les changements de mentalité en cours : l'auteur ne fait aucune mention du monarque comme « bon père des Français $»{ }^{69}$, n'insère aucun plaidoyer ou réplique pour l'innocenter ou le dis-

(67) Voir aussi André Tissier, op. cit., p. 173.

(68) Sur l'un des manuscrits, les vers « Obéir à ses rois, ou servir sa patrie/ Pour tous deux à la fois sacrifier sa vie » (p. 22 de l'édition) sont remplacés sur becquet par « Combattre les tirans et servir la Patrie, / pour elle dans tous temps sacrifier sa vie » (f. 232 r). De même, la tirade du Grenadier «>Grand roi! Des cœurs pervers, de lâches courtisans / Voudraient d'un joug chéri nous peindre impatients; / Nous offrir méprisant les pouvoirs les plus justes; / Renversant sans respect les lois les plus augustes; / Ils égarent ton cœur. C'est contre eux aujourd'hui / Que ton juste corroux doit chercher un appui. / Reviens de ton erreur; vois ce peuple fidèle, / Brûlant pour lui d'amour, plein de crainte \& de zèle. / Vois le fier, mais soumis; volage, mais constant, / Et vois dans ce transport, l'écho du sentiment $!<»$ (p. 36 de l'édition) est supprimée et remplacée sur becquet par un simple «Au bien de la patrie » (f. 213 v) et « À notre Nation » (f. 240 r).

(69) Les pièces du corpus traduisent encore le sentiment des contemporains; ainsi que le souligne, entre autres, Lynn Hunt, à partir de juin 1791 et pendant les mois suivants, l'iconographie aussi présente une figure du roi de plus en plus ridiculisée et dénuée des marques de la royauté : Lynn Hunt, Le roman familial de la Révolution française, Paris, Albin Michel, 1995, p. 59 sq. 
tinguer des aristocrates conspirateurs, et affiche finalement une neutralité idéologique ambiguë et peu efficace.

Malgré les réécritures et les révisions, ces pièces trouvent de moins en moins de place dans les répertoires, et d'autres textes sur le sujet ne sont pas acceptés. C'est le cas, notamment, de Les deux Prisonniers ou la Fameuse Journée (1792), une pièce bien singulière et qui mérite quelques reflexions ${ }^{70}$. Auteur d'ouvrages représentés à l'Opéra, Martin affiche dans sa préface des positions quelque peu différentes de celles mentionnées dans les autres paratextes. D'un côté, l'auteur suit la route déjà tracée et souligne la véracité d'un « drame historique et lyrique » dont il essaie de valider les deux «volets »- le séjour à la Bastille de Jean Henri, dit Danry, dit Masers de Latude et de son compagnon d'Alègre, et la prise de la forteresse - en s'appuyant sur des sources célèbres - les « Mémoires de M. de Latude, l'Ouvrage de M. Linguet sur la Bastille, les Lettres de Cachet, les Prisons d'État de Mirabeau, et la Brochure dans laquelle M. Dusaulx a tracé, avec autant d'énergie que de précision, les grands événéments qui ont décidé la Révolution » (p. 2) - dont il reprend des formules ou des passages ${ }^{71}$ ainsi que des épisodes célèbres à la charge émotionnelle et pathétique importante $^{72}$. De l'autre, il entreprend une opération de circonstance qu'il veut «pour ainsi dire, indépendante des circonstances » (p. 10) et dégagée de tout esprit de parti. Pour ce faire, Martin se détache des assertions d'adhésion totale au fait historique qui constituaient le leitmotiv des

(70) L'ouvrage retrace l'évasion de Latude et de son compagnon d'Alègre comme si elle avait eu lieu le 13 juillet 1789 et non en 1756, permettant de la sorte à l'auteur de faire participer les deux prisonniers à la célèbre journée.

(71) Par exemple, on retrouve le "ô siecle ô mémoire » repris par Dusaulx dans son ouvrage, ainsi qu'un épisode cité par celui-ci concernant un jeune assiégeant qui déguise son père pour le faire sortir de la forteresse où il faisait partie de la garnison, voir Dusaulx, op. cit., p. 48. Encore, tout le passage pathétique suivant est entièrement copié de l'ouvrage de Linguet : " sentir que l'on a sur sa tête ou sous ses pieds un être malheureux, à qui l'on pourroit donner, ou de qui l'on pourroit recevoir du soulagement; l'entendre marcher, soupirer; penser qu'on n'en est éloigné que d'une demi-toise ; combiner sans cesse le plaisir de franchir cet espace et l'impossibilité d'y réussir; avoir également à s'affliger, et du fracas qui annonce un nouveau venu, condamné à partager vos fers, sans les alléger, et du silence de ces cachots, qui vous avertit qu'un des compagnons de votre misere a été plus fortuné que vous, c'est un tourment [supplice dans les Mémoires] dont on ne peut pas se former d'idée. »( p. 18), voir M. Linguet, Mémoires sur la Bastille, Londres, Spilsbury, 1783 , p. 62-63.

(72) Notamment sont évoqués et présentés sur scène non seulement la fameuse échelle, mais aussi, par exemple, la flûte bien mentionnée par Latude dans ses Mémoires, ainsi que d'autres épisodes de vie quotidienne. Voir Le Despotisme dévoilé, ou Mémoires de Henri Masers de Latude, détenu pendant trente-cinq ans dans diverses prisons d'État, rédigés sur les pièces originales par M. Thiéry, Paris, Lejay fils, 1790, 3 vols. 
autres paratextes : le télescopage affiché et trop évident entre histoire l'évocation de la prise de la forteresse -, mémoires - l'emprisonnement de Latude - et fiction - la participation de celui-ci au siège - relève de la recherche d'un effet théâtral basé sur l'illusion, sur l'invention dramatique, sur la nécessité et l'importance d'une licence poétique qui réaffirme le rôle du dramaturge par rapport à celui de l'historien/témoin. Souvent sacrifiés sur l'autel de l'authenticité, l'imagination et l'art dramatique semblent ici récupérer du terrain puisque Martin soumet ses personnages « aux événements qui sont réellement arrivés » tout en voulant les faire ressembler à des personnages de fiction, à savoir à « tous les Héros de Tragédie, lesquels agissent et parlent au gré de l'Auteur [...] non seulement tels qu'ils ont été, mais encore tels qu'ils se seroient montrés dans telles ou telles circonstances données » (p. 9). Dans la préface, la dignité des « fictions poétiques » est d'ailleurs rétablie par un auteur qui s'abrite derrière les mots de Boileau et la tradition littéraire pour justifier son écart fictionnel («Inventez des ressorts qui puissent m'attacher $[\ldots]$ c'est en vertu de ce précepte qu'il est permis aux Poetes d'intervertir l'ordre des temps, de rapprocher les événements, de les tronquer même à leur gré », p. 8). Sa revendication d'une esthétique dramatique plus classique porte aussi sur le respect de préceptes aristotéliciens négligés dans d'autres textes ${ }^{73}:$ les règles d'unité, la nécessité de l'achèvement et de la cohérence du texte, le choix de ne représenter que la «nature peinte en beau, la nature choisie» (p. 10), écartant de la sorte l'évocation de détails sinistres et choisissant d'insérer dans la représentation d'un sujet patriotique et glorieux une histoire d'amour susceptible de rencontrer toutes les faveurs - argument, celui-ci, déjà utilisé par Voltaire dans la préface de Zä̈re.

Il s'agit bien sûr d'une opération commerciale visant le renouvellement d'un sujet assez rebattu : l'auteur souligne l'originalité de sa représentation en musique par rapport à « des pantomimes de siège, et autres accessoires, dont il faut être très sobre, pour obtenir un succès durable » (p. 11), exploite la valeur symbolique de l'événement ainsi que l'engouement populaire pour le culte des « reliques » et pour la figure de

(73) Brizard affiche de la sorte son insouciance à l'égard de l'esthétique classique : « Point d'unité de tems, point d'unité de lieu; elles étaient impossibles, ou les événements altérés [...] auroient fait accuser notre Ecrivain de sacrifier la vérité aux règles gênantes de l'éloquence » (p. 3-4); de même, David réclame de l'indulgence pour un ouvrage qui présente « des fautes contre les règles de l'art dramatique, \& peu de connaissance du théâtre » mais estime que « le public souffrira [...] plus aisément que je me sois écarté des principes de l'art pour suivre la vérité, qu'il ne me pardonnerait de m'être écarté de la vérité pour suivre l'art exactement » (p. 3-4). 
Latude, doublement acclamée en qualité de « victime des rois, victime de la Bastille et des ordres arbitraires ${ }^{74}$ et en tant qu'apôtre courageux d'une liberté fort recherchée, ainsi qu'en héros presque mélodramatique et bien moins politique que le Citoyen-Soldat Harné. Apparemment, ses efforts ne suffisent pas à renouveler des enthousiasmes déjà mitigés. Touffue et peu vraisemblable, la pièce n'est pas représentée. Pour nous, elle s'avère toutefois intéressante en ce qu'elle constitue une autre tentative, esthétique et idéologique, de révision de l'événement. Pendant un moment politiquement fort délicat, cette opération fictionnelle - « S'il [Latude] se fut évadé en 1789 au lieu de 1756, il étoit possible qu'il lui arriva ce que j'ai supposé à son égard » (p. 9) - permet à Martin de restituer une prise de la Bastille hypothétique et surtout domestiquée, l'incursion dans le domaine des possibles légitimant bien d'autres fictions : la pièce s'achève donc sur la dimension festive, accrue et légitimée par la fête de mariage, d'un événement rapporté non par les assiégeants réels - tel le grenadier Harné - mais par des personnages susceptibles d'intéresser le public - Latude ou l'hôte Père Duchesne, parti de sa campagne libérer les prisonniers avec les autres paysans - qui finalement n'ont pas vécu les inquiétudes et les bouleversements parisiens précédant et suivant la prise. Lélément fictionnel permet à l'auteur de relire l'événement au rabais, d'en exploiter la valeur symbolique et patriotique en escamotant toute interprétation idéologique plus étendue, toute signification politique troublante, puisque les paysans et surtout Latude rentrant juste après le siège ne sont tenus de connaître ni les massacres ni la réalité d'un complot dont ils peuvent bien légitimement n'avoir eu que des échos fort vagues.

Finalement peu efficace, cette tentative d'une solution dramatique différente témoigne d'une autre expérience de révision d'un événementsymbole à la dramatisation délicate et dont la réception s'avère - ainsi que celle de maintes pièces d'actualité - fort politisée et soumise aux mutations rapides d'un esprit public tout puissant. Pendant une période où le politique investit de façon tellement importante un événement dramatique plus que jamais soumis aux aléas socio-politiques, l'analyse de la réception des pièces et de leur présence ou absence dans les répertoires peut nous éclairer non seulement sur l'histoire du théâtre, mais aussi et surtout sur l'histoire de la Révolution et de l'évolution des mentalités,

(74) Frantz Funck-Brentano, Introduction à Mémoires authentiques de Latude, Paris, Fayard, 1795 , p. 39. 
ainsi que le rappelle Sophie Marchand quand elle écrit que «l'histoire du théâtre de ce temps est moins une histoire des œuvres qu'une histoire des crises $\gg{ }^{75}$.

Paola Perazzolo

Université de Vérone ANR THEREPSICORE Centre d'Étude de la Langue et de la Littérature françaises des XVII ${ }^{\mathrm{e}}$ et $\mathrm{XVIII}{ }^{\mathrm{e}}$ siècles (CELLF $17^{\mathrm{e}}-18^{\mathrm{e}}$ siècles, Université Paris IV) paola.perazzolo@univr.it 